\title{
Micro-Environmental Systems and Endothelial Cells in Cooperative Tumorigenesis Account for Potential Malignant Transformation in Neurofibromatosis Type 1 Patients
}

\author{
Lawrence M Agius* \\ Department of Pathology, University of Malta Medical School, Europe
}

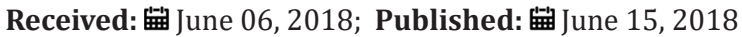

*Corresponding author: Lawrence M Agius, Department Of Pathology, Mater Dei Hospital, Tal-Qroqq, University of Malta Medical School, Msida, Europe

\begin{abstract}
Overall tumorigenesis in neurofibromatosis type 1 patients constitutes a series of specific targeting events with a central role enacted by proliferation of fibroblasts and endothelial cells in overproduction of growth factors and cytokines such as transforming growth factor-beta and CXCL12 cytokine. The plexiform neurofibroma well-illustrates dimensions of such cooperative participation within operative fields of the initial Schwann cell proliferation leading in a significant number of patients to malignant transformation of the peripheral nerve sheath tumors. Inclusive directions in operative targeting of Schwann cells or astrocytes are staged performance in the transformation of hyperproliferative induction and constitute further evolutionarily defined incorporation of such systems as endothelial cells. Hyperproliferative cell subsets are initial and also consequential target formulation of potential malignant states as induced in malignant peripheral nerve sheath tumors.
\end{abstract}

\section{Introduction}

Neurofibromatosis type 1 (NF1) is a neurogenetic disorder and involves both heterozygous and homozygous absence/reduction of neurofibromin that acts normally as a tumor suppressor. There is a need to assess predisposing genetic factors and loss of heterozygosity causing emergence of aggressive neoplasms in patients with NF1 [1]. The two hit hypothesis helps account for the emergence of Schwann cell-based proliferations and for neurofibromas and plexiform neurofibromas. Gherkin may act on tumorigenesis of cutaneous neurofibromas via growth hormone secretagogue receptor [2]. It is important to consider the neurofibroma that is based on micro-environmental potentiation of tumor generation in patients that develop malignant nerve sheath tumors and astrocytomas in patients with NF1 +/- genotype; this occurs in a manner that involves growth factor overactivity and mast cell and endothelial overactivity within a milieu that dysfunctionally stimulates tumorigenesis. Reactive oxygen species overproduction lead to epithelial-mesenchymal transit in patients with neurofibromin deficiency and plays a crucial role in NF1 tumor growth [3]. RAS activation alone is not sufficient for malignant transformation of peripheral nerve sheath tumors; signal transduction may potentially help identify therapies for this neoplasm type [4].

\section{Neurofibromin}

The dynamics of neurofibromin as a cytoplasmic protein involve the regulation of K-Ras, and the PI3K/Akt pathways; absence of neurofibromin leads to overactivation of these pathways in various ways in inducing tumorigenesis in such lesions as optic tract pilocytic astrocytomas, brain stem astrocytomas and also other CNS astrocytomas in terms of progression of these lesions. The cell of origin determines the temporal course of neurofibromatosis-1 low-grade glioma formation [5]. The micro-environment of plexiform neurofibromas of peripheral nerves and of nerve plexi include a $10 \%$ risk of malignant change with subsequent aggressive clinical behavior in the affected patients. Over expression of cellular retinoid acid binding protein 2 is reported in several cancer types, including malignant peripheral nerve sheath tumors (MPNSTs) [6]. 


\section{Related Tumor Predispositions}

The neurofibromin insufficiency status in Schwann cells and fibroblasts allows for enhanced participation of immune system component cells such as microglia as evidenced in optic pathway low-grade astrocytomas. Telomere erosion is described in many tumor types and may potentially drive genomic instability and clonal progression in NF1-associated MPNSTs [7]. Tumor dimensions include proliferation of astrocytic cells in optic pathways, and of various subtypes of stromal cells such as fibroblasts and mast cells in the peripheral nervous system. It is significant to consider particularly the micro-environmental active participation in the genesis of the most common tumor type in Neurofibromatosis type 1 patient, that is the neurofibroma, which invokes proliferation of fibroblasts and endothelial cells. The congenital plexiform neurofibroma is in fact a hypervascular lesion that transgresses tissue margins and induces a significant risk for malignant transformation. NF1 loss is the primary driver of tumorigenesis in neurofibromatosis type 1-related plexiform neurofibroma [8]. It is further to such considerations that important cooperative intervention in malignant transformation of plexiform neurofibromas invokes multi-type cells in inducing proliferation of an integral Schwann cell-fibroblastic twin population in enhancing potential malignant transformation of the peripheral nerve sheath. A therapeutic window for neuroprotective intervention exists as detected by optical coherence tomography in mice with optic glioma, and particularly as an accurate biomarker of retinal ganglion cell apoptosis [9]. The heterozygous absence of one neurofibromin allele in mice results in plexiform neurofibromas and low-grade optic pathway astrocytomas. Mast cells appear to play a causal role in neurofibroma formation and also in microglia in optic pathway glioma evolution [10]. Such implications of the micro-enviromental factors includes a distinctive cooperative participation that carries implications for significant enhancement of cell proliferation and of such cytokines such as transforming growth factor and CXCL12 in formulating malignant transformation in such tumors. The methylemetetrahydrofolate reductase 1298 and 677 gene polymorphisms are related to optic glioma and hamartoma risk in NF1 patients through effects on DNA synthesis and methylation [11].

\section{Convergent Targeting}

The related tuberous sclerosis complex is analogous to neurofibromatosis type 1 as a neurogenetic disorder associated with increased risk for astrocytomas in the form of subependymal giant cell astrocytomas. A convergent targeting of systems of cell proliferation include in particular cyclic AMP and Ras in a manner that includes dimensions of micro-environmental conditioning. Mutations of the NF 1 gene are frequent in many cancer types in patients without NF1 and this is suggestive of a more general role for the NF1 gene in oncogenesis. In melanoma NF1 mutations potentially drive tumorigensis and promote drug resistance [12] Inclusive dynamics allow for permissive tumorigenesis in a manner that includes the incorporation of malignant transformation within confines of a Schwann cell-fibroblast-endothelial cell system in the case of malignant peripheral nerve sheath tumors. Astrocytes and microglia are analogous counterparts in the induction of CNS astrocytomas. Such considerations are inclusive phenomena of multi-component induction of potential malignancy that recharacterizes conditioning of the micro-environment of proliferative states preceding tumorigenesis. Interaction between neoplastic Schwann cells and their surrounding neural microenvironment has important implications for early cellular events promoting tumorigenesis in neurofibroma development [13].

\section{Performance Dynamics}

Performance dynamics of tumors in neurofibromatosis type 1 may potentially modify the biologic significance of a two-hit hypothesis in a manner that implicates micro-environmental conditioning of the resultant cell hyperplasias and proliferations in such lesions as peripheral nerve sheath tumors and astrocytomas. NF1 provides unique vantage points to examine co-contributions of molecular, cellular, and tissue processes in tumor biology [14]. Such proposed dimensions invoke in particular an over-activation in production and action of growth factors that provoke selective malignant transformation of hyper-proliferative lesions composed of Schwann cells and astrocytes in the peripheral and central nervous systems respectively. Plasma soluble levels of transforming growth factor-beta and interleukin- 6 are increased in NF1 patients and a shift towards an anti0inflammatory profile has been reported in cells expressing cytokines [15].

\section{Hyperproliferation}

The hyperproliferative states affecting Schwann cells and astrocytes invoke also fibroblast and microglial cell proliferations in a manner transforming tumorigenesis. Such facilitation to tumorigenesis invokes dimensions of transformation as well seen in plexiform neurofibromas that may undergo malignant transformation in a significant number of affected individuals. Such considerations are selective targeting of specific cell subpopulations in a manner that allows permissive transformation. Insertional mutagenesis identifies a STAT3/Arid1b/beta-catenin pathway that drives neurofibroma initiation in the context of Nf1 loss [16]. Mast cells and fibroblasts may potentially incorporate endothelial cells that may participate as central dysregulatory dimensions in plexiform neurofibroma tumorigenesis. The provocations for malignant transformation further cooperate in systems of derivative consequence as hypervascular lesions that subsequently lead to potential malignant cells in individual patients. Cross species comparative oncogenomic may identify driver mutations in mouse cancer models and allow validation in human tumors [17].

\section{Concluding Remarks}

Propositional implications in tumorigenesis include the multi-component participation of Schwann cells on the one 
hand and of fibroblasts, mast cells, endothelial cells and also of microglia in an inductive process that includes specific pathways of malignant transformation. Endothelial cell proliferation is related to substantial participation in modes related to key-events of increased proliferation of Schwann cells and astrocytes in initial stages of lesion infliction. Inclusive phenomena have thus become systems of consequence in affecting such specific cell proliferative states. Such events occur within the added dimensions of directed targeting of multiple-agent micro environmental modeling of the initial proliferation of the Schwann cells or astrocytes. A pivotal series of roles played by fibroblasts, endothelial cells, mast cells and of microglia and astrocytes appears a dynamic milieu within added consequences of malignant transformation of both Schwann cells and astrocytes that progress as cooperative systems of tumorigenesis.

\section{References}

1. Saurez Kelly LP, Akagi K, Reeser JW, Samorodnitsky E, Reeder M, et al. (2018) Metaplastic breast cancer in a patient with neurofibromatosis type 1 and somatic loss of heterozygosity. Cold Spring Harb Mol Case Stud 4(2).

2. Rozza-de Menezes RE, Gaglionone NC, Andrade Losso RM, Siqueira OHK, Almeida LM, et al. (2017) Receptor of ghrelin is expressed in cutaneous neurofibromas of individuals with neurofibromatosis 1 . Orphanet J Rare Dis 12(1): 186 .

3. Zhang Y, Zhou R, Qu Y, Shu M, Guo S, et al. (2017) Lipoamide inhibits NF1 deficiency-induced epithelial-mesenchymal transition in murine Schwann cells. Arch Med Res 48(6): 498-505.

4. Kim A, Pratilas CA (2018) The promise of signal transduction in genetically driven sarcomas of the nerve. Exp Neural 299(Pt B): 317 325.

5. Solga AC, Toonen JA, Pan Y, Cimino PJ, Ma Y, et al. (2017) The cell of origin dictates the temporal course of neurofibromatosis-1 (Nf1) low-grade glioma formation. Oncotarget 8(29): 47206-47215.

6. Fischer Huchzermeyer S, Dombrowski A, Hagel C, Mauten VF, Schittenhelm J, et al. (2017) The cellular retinoid acid binding protein 2 promotes survival of malignant peripheral nerve sheath tumor cells. Am J Pathol 187(7): 1623-1632.

7. Jones RE, Grinstead JW, Sedani A, Baird D, Upadhyay M (2017) Telomere erosion in NF1 tumorigenesis. Oncotarget 8(25): 40132-40139.

8. Pemov A, Li H, Patidar R, Hansen NF, Sindiri S, et al. (2018) The primacy of NF1 loss as the driver of tumorigenesis in neurofibromatosis type 1-associated plexiform neurofibromas. Oncogene 36(22): 3168-3177.

9. Toonen JA, Ma Y, Gutmann DH (2017) Defining the temporal course of murine neurofibromatosis-1 optic gliomagenesis reveals a therapeutic window to attenuate retinal dysfunction. Neuro Oncol 19(6): 808-819.

10. Karmakar S, Reilly KM (2017) The role of the immune system in neurofibromatosis type 1-associated nervous system tumors. CNS Oncol $6(1): 45-60$

11. Tanyildiz HG, Yesil S, Bozkurt C, Candir MO, Akpinar Tetgunduz S, et al. (2016) Are the methylemetetrahydrofolate reductase 1298 and 677 gene polymorphisms related to optic glioma and hamartoma risk in neurofibromatosis type 1 patients?. Turk J Podiatry 58(2): 152-158.

12. Peltonen S, Kallionpaa RA, Peltonen J (2017) Neurofibromatosis type 1 (NF1) gene: beyond cafe au last spots and dermal neurofibromas. Exp Dermal 26(7): 645-648.

13. Liao CP, Pradhan S, Chen Z, Patel AJ, Booker RC, et al. (2016) The role of nerve microenvironment for neurofibroma development. Oncotarget 7(38): 61500-61508.

14. Rubin JB (2015) Intersections at the crossroads: Neurofibromatosis type 1, cAMP, sex, and glioma risk. Mol Cell Oncol 3(3).

15. Torres KC, Lima G, Simoes E Silva AC, Lubambo I, Rodrigues LO, et al. (2016) Immune markers in the RASopathy neurofibromatosis type 1.$]$ Neuroimmunol 295-296: 122-129.

16. Wu J, Keng VW, Patmore DM, Kendall JJ, Patel AV, et al. (2016) Insertional mutagenesis identifies a STAT3/Arid1b/beta-catenin pathway driving neurofibroma initiation. Cell Rep 14(8): 1979-1990.

17. Carroll SL (2016) The challenge of cancer genomics in rare nervous system neoplasms: malignant peripheral nerve sheath tutors as a paradigm for cross-species comparative oncogenomics. Am J Pathol 186(3): 464-477.
(C) (P) This work is licensed under Creative Commons Attribution 4.0 License To Submit Your Article Click Here: Submit Article

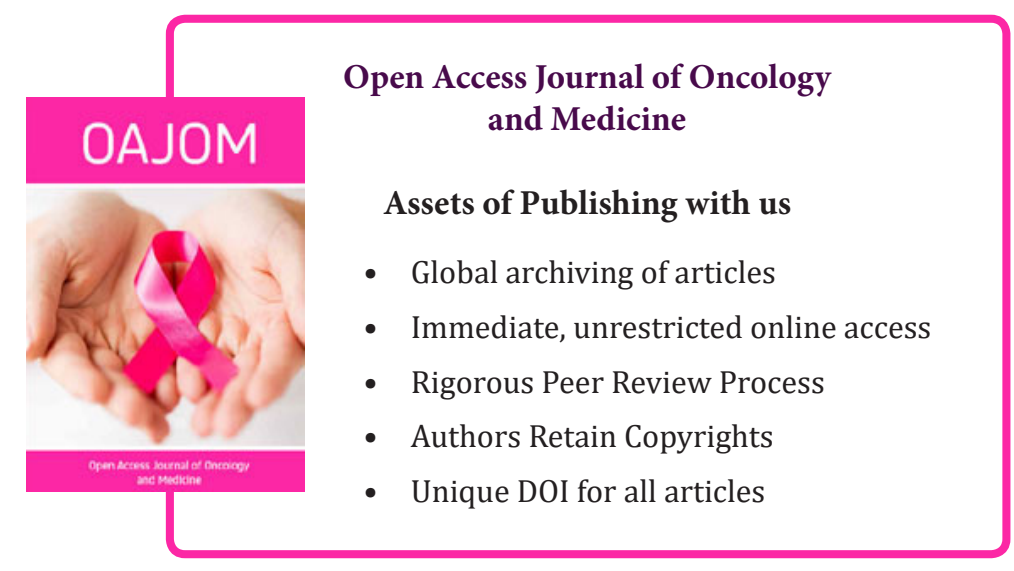

